Research Paper

\title{
N6-methyladenosine-related Genomic Targets are Altered in Breast Cancer Tissue and Associated with Poor Survival
}

\author{
Liwen Liu ${ }^{1,2^{*}}$, Xin Liu ${ }^{1,2^{*}}$, Zihui Dong ${ }^{1,2}$, Jianhao Li1 ${ }^{1,2}$, Yan $\mathrm{Yu}^{1,2}$, Xiaolong Chen ${ }^{1,2}$, Fang Ren², Guangying \\ Cui ${ }^{1,2}$, Ranran Sun ${ }^{1,2,3 凶}$ \\ 1. Precision Medicine Center, The First Affiliated Hospital of Zhengzhou University, Zhengzhou 450052, China \\ 2. Key Laboratory of Clinical Medicine, The First Affiliated Hospital of Zhengzhou University, Zhengzhou 450052, China \\ 3. National Engineering Laboratory for Internet Medical System and Application, The First Affiliated Hospital of Zhengzhou University, Zhengzhou 450052, \\ Henan, China \\ *These authors contributed equally to this work. \\ $\triangle$ Corresponding author: sunran1986318@163.com (Ranran Sun)
}

(c) The author(s). This is an open access article distributed under the terms of the Creative Commons Attribution License (https://creativecommons.org/licenses/by/4.0/). See http://ivyspring.com/terms for full terms and conditions.

Received: 2019.03.19; Accepted: 2019.08.12; Published: 2019.08.29

\begin{abstract}
Purpose: The ectopic expression of N6-methyladenosine (m6A) associated genes is a common feature of multiple tumors. However, little is known about the expression status and the prognostic value of these genes in human breast cancer (BRC). Herein, we conducted a comprehensive analysis to identify the expression profiling and clinical significance of m6A-related genomic targets in BRC.

Materials and Methods: The expression data including 1109 BRC tissues and 113 normal breast tissues were obtained from The Cancer Genome Atlas (TCGA) database to evaluate the mRNA expression levels of m6A-related genomic targets. In addition, 6 independent BRCA cohorts retrieved from the Gene Expression Omnibus (GEO) database were enrolled to further ascertain the expression profiling of m6A-related genomic targets. Meanwhile, the immunohistochemical (IHC) staining data from BRC tissue microarray (TMA) cohort and the Human Protein Atlas (HPA) database were used to evaluate the proteomic expression of m6A-related genomic targets. Immunofluorescence (IF) analysis was performed to validate the subcellular location of m6A-related genomic targets. Moreover, the prognostic value of m6A-related genomic targets in BRC was analyzed by Kaplan-Meier analysis and Cox regression models.
\end{abstract}

Results: m6A-related genomic targets were differentially expressed in BRC tissues. TMA IHC staining showed that most of the m6A-related genomic targets were significantly altered at the protein level (either upregulated or downregulated), consistent with their changes in the genomic profile. IF analysis showed the subcellular location of m6A-related genomic targets in BRC cell lines. Furthermore, we demonstrated that overexpression of YTHDF1 $(P=0.049)$, YTHDF3 $(P<0.001)$ and KIAA1429 $(P=0.032)$ predicted poor prognosis in terms of overall survival (OS). Upregulation of YTHDF3 was an independent prognostic factor for OS in patients with BRC $(\mathrm{P}=0.036)$.

Conclusion: m6A-related genomic targets are significantly altered in BRC and predict poor prognosis. These m6A-related genomic targets could serve as novel prognostic biomarkers for BRC.

Key words: N6-methyladenosine, Breast cancer, YTHDF3, Prognosis, Biomarkers.

\section{Introduction}

Breast cancer (BRC) is the most prevalent malignancy with a heterogeneous group of molecular subtypes [1]. BRC is also the leading cause of cancer-related death for women in the vast majority countries, with an estimated 2.1 million newly diagnosed BRC cases and 626 thousands deaths 
predicted in 2018, accounting for almost $25 \%$ cancer cases and $6.6 \%$ cancer deaths among women worldwide [2, 3]. Despite a number of available treatment strategies, the prognosis of BRC remains poor. Hence, identifying additional genomic targets for the early diagnosis and effective treatment of BRC is an ever-increasing need.

Epigenetic dysregulation is a consistent feature in multiple cancers [4]. In addition to the well-known classical epigenetic modulating mechanisms, such as chromatin remodelling [5], DNA methylation [6] and histone modifications [7], RNA N6-methyladenosine (m6A) has an emerging recognized role as an epigenetic regulator. $\mathrm{m} 6 \mathrm{~A}$, a prevalent internal modification in mammalian mRNAs and noncoding RNAs, has emerged in a ubiquitous role to fine tune RNA processing by acting as "writers", "erasers" and "readers" [8]. Through this special approach, m6A provides a new mechanism of epigenetic regulation in many bioprocesses such as meiosis [9], sex determination [10], neural development [11], cardiac rhythms [12] and stress response [13]. Interestingly, $\mathrm{m} 6 \mathrm{~A}$ is also required for some human diseases, and recent publications in the cancer research field have provided new insights into m6A. Multiple studies have identified that several m6A-related molecules are involved in mutagenesis and carcinogenesis [14-16]. However, little is known about the critical role of m6A-related genomic targets in BRC.

In this study, we identified a class of m6A-related genomic targets expression profiles in BRC based on The Cancer Genome Atlas (TCGA) and the Gene Expression Omnibus database (GEO). In addition, immunofluorescence (IF) staining and tissue microarray (TMA) analysis were performed to detect subcellular locations and expression patterns of m6A-related genomic targets at the protein level. Furthermore, we also evaluated the robustness of m6A-related genomic targets dysregulation in patients through survival analysis to explore the potential value of m6A in BRC. Our findings suggest that m6A-related genomic targets could serve as novel prognostic biomarkers for BRC.

\section{Materials and Methods}

\section{Expression data sets}

The TCGA-BRCA cohort data of 1109 BRC patients and 113 normal patients were downloaded from The Cancer Genome Atlas (TCGA, https://tcga-data.nci.nih.gov/tcga/). 6 independent cohorts, including GSE70947, GSE15852, GSE109169, GSE36295, GSE29044 and GSE24124, were obtained from the Gene Expression Omnibus (GEO https://www.ncbi.nlm.nih.gov/geo/) database.
These datasets were used to analyse the expression profiles of m6A-associated molecules in BRC and evaluate their correlations with the clinical prognosis.

\section{Cell lines and cell culture}

Human BRC cell lines MDA-MB-231, MDA-MB-468 and MCF-7 were purchased from ATCC (Manassas, USA). Cells were maintained at $37^{\circ} \mathrm{C}$ in a humidified atmosphere of $5 \% \mathrm{CO}_{2}$ in Dulbecco's modified Eagle medium (DMEM) supplemented with $10 \%$ fetal bovine serum (Gibco, New York, NY, USA) and 100 U/mL penicillin/streptomycin (Corning, New York, NY, USA).

\section{TMA cohorts}

The tissue microarray (TMA) containing 20 BRC specimens and 20 normal breast tissue specimens was obtained from the First Affiliated Hospital of Zhengzhou University. The Institutional Review Board of the First Affiliated Hospital of Zhengzhou University approved this study. And informed consent for this study was collected from all participants. To further confirm the expression of m6A-related genes, we analysed another BRC TMA cohort obtained from the Human Protein Atlas (HPA, https://www.proteinatlas.org/) database.

\section{Immunohistochemical staining}

TMA sections $(5 \mu \mathrm{m})$ were deparaffinized, hydrated, blocked for endogenous peroxidases and antigen retrieval. After blocking for an hour at room temperature, the slide was incubated with primary antibody at $4^{\circ} \mathrm{C}$ overnight. And then samples were probed with biotinylated goat anti-rabbit secondary antibody. Finally, the slide was detected by SignalStain ${ }^{\circledR}$ DAB (Cell Signaling Technology, Danvers, MA) and counterstained with haematoxylin QS (Vector Laboratories). Cells containing brown granules were independently counted by two pathologists who were blinded to clinical parameters, and the samples were scored according to the proportion of positive cells as follows: 0 , none; 1 , $<25 \% ; 2,25 \%-50 \% ; 3,51 \%-75 \%$; and $4,76 \%-100 \%$. The staining intensity was scored as follows: 0 , none; 1 , weak; 2, moderate; and 3, strong. The total staining score (range $0-12$ ) was calculated by multiplying the two subscores, and the samples with scores of 0-3, 4-6 and 9-12 were respectively classified as low expression, moderate expression and high expression.

\section{Immunofluorescence assay}

For immunofluorescence (IF) analysis, the BRC cells cultured in 24-well plate were fixed with $4 \%$ paraformaldehyde (PFA) in PBS for $20 \mathrm{~min}$ at room temperature. And then cells were permeabilized with 
0.5\% Triton X-100 (Solarbio, Shanghai, China) in PBS. After blocking with $1 \%$ bovine serum albumin (BSA), we incubated the cells with primary antibody at $4^{\circ} \mathrm{C}$ overnight and then the appropriate corresponding secondary antibodies (Jackson ImmunoResearch Inc., USA) at room temperature for $30 \mathrm{~min}$. Finally, the nuclei were counterstained with DAPI (Beyotime, China), and images were obtained with a Zeiss Axio microscope (Zeiss, Oberkochen, Germany). The detailed information of antibodies used in this study is listed in table $S$.

\section{Statistical analysis}

Statistical analysis was performed using SPSS software (version 23.0, Inc., Chicago, IL) and GraphPad Prism 6 software (GraphPad Software, Inc., La Jolla, CA, USA). The differences between two independent groups were analysed using Student's $t$ test (unpaired, two-tailed). For each significantly ectopically expressed molecule, a Kaplan-Meier overall survival and relapse-free survival analysis were performed with a log-rank test. Cox regression analysis of univariate and multivariate was performed to ascertain independent factors. A $\mathrm{P}<0.05$ was considered statistically significant.

\section{Results}

\section{Distinct differential expression profiles of m6A-related genomic targets in BRC}

To gain a holistic insight into the m6A-related genomic aberrations underlying BRC, we downloaded RNA transcriptomic datasets containing next generation sequencing (RNA-seq) data of 1109 BRC tissues and 113 non-tumor tissues from TCGA project (TCGA-BRCA). We analyzed the mRNA expression levels of the known m6A-related genomic targets including m6A "writers", such as WTAP (Wilms' tumour 1-associated protein), RBM15, KIAA1429, METTL3, METTL14, METTL16 and RBM15B, m6A "readers", such as YTHDF1, YTHDF2, YTHDF3, YTHDC1, HNRNPC and HNRNPA2B1, and m6A "erasers", such as FTO and ALKBH5 (Figure 1A). The results showed that a considerable number of m6A-related genomic targets were differentially expressed in BRC tissues in comparison with those in normal breast tissues (Figure 1A). Of these genes, 6 genes such as KIAA1429 $(\mathrm{P}<0.001)$, RBM15 $(\mathrm{P}<0.01), \quad$ YTHDF1 $(\mathrm{P}<0.001), \quad$ YTHDF2 $(\mathrm{P}=0.022), \mathrm{HNRNPC}(\mathrm{P}<0.001)$ and HNRNPA2B1 $(\mathrm{P}<0.001)$ were upregulated and 5 genes such as WTAP $(\mathrm{P}<0.001), \quad$ METTL14 $(\mathrm{P}<0.001), \quad$ METTL16 $(\mathrm{P}<0.001)$, YTHDC1 $(\mathrm{P}=0.013)$ and FTO $(\mathrm{P}<0.001)$ were downregulated in BRC tissues, while others were not significantly different. Furthermore, we validated the expression of these aberrant m6A-associated genomic targets in 6 independent BRCA GEO datasets with microarray platforms (Table 1). Notably, GEO dataset analysis showed that the m6A-related genomic targets exhibited similar expression patterns in BRC (Figure 1B). Taken together, these data indicate a strong deregulation of several m6A-related genomic targets in human BRC and show that these alterations are broadly consistent across clinical cohorts.

Table 1. GEO Microarray Data enrolled in to Identify Altered m6A Targets in Breast cancer

\begin{tabular}{llllll}
\hline Accession number & Platform & \multicolumn{2}{l}{ Number of samples } & Country & Years \\
\cline { 3 - 4 } & & Non-tumor & Breast cancer & & \\
\hline GSE70947 & Agilent & 148 & 148 & USA & 2016 \\
GSE15852 & Affymetrix & 43 & 43 & Malaysia & 2009 \\
GSE109169 & Affymetrix & 25 & 25 & Taiwan & 2018 \\
GSE29044 & Affymetrix & 36 & 73 & Saudi Arabia & 2014 \\
GSE24124 & Agilent & 20 & 99 & Taiwan & 2010 \\
GSE36295 & Affymetrix & 5 & 45 & Saudi Arabia & 2014 \\
Total & & 277 & 433 & & \\
\hline
\end{tabular}

\section{Altered m6A-related genomic targets show similar expression patterns at the protein level}

TMA data from the ZZU cohort and the Human Protein Atlas (HPA) database were further analyzed to validate the expression patterns of altered m6A-related genomic targets in BRC tissues and noncancerous breast tissues at the protein level. First, we examined the expression levels of m6A "writers" in BRC tissue microarrays comprising 20 BRC specimens and adjacent normal specimens. Immunohistochemistry (IHC) staining analysis indicated the significant upregulation of WTAP $(\mathrm{P}=0.002)$, KIAA1429 $(\mathrm{P}<0.001)$ and RBM15 $(\mathrm{P}=0.012)$ in BRC specimens (Figure $\mathbf{2 A}$ and $\mathbf{2 B}$ ). While the corresponding mRNA expression level of WTAP was lower in BRC tissues, the protein expression levels of KIAA1429 and RBM15 were consistent with their transcriptional levels in comparison with those in the adjacent normal tissues. In contrast, we did not detect any significant changes in protein expression levels of METTL3, METTL14, METTL16 or RBM15B between BRC and adjacent normal tissues (Figure 2A and 2B). Moreover, IHC staining results obtained from the HPA database also demonstrated the similar expression patterns of m6A "writers" in BRC, consistent with their mRNA levels (Figure 3A-F). Altogether, these results further confirmed the highly significant dysregulation of m6A "writers" in BRC.

In addition to m6A "writers", we also analyzed the expression of m6A "readers" in BRC tissues. IHC staining results showed that the majority of genes associated with m6A "readers" displayed markedly differential expression in BRC TMA. While the 
expression of YTHDC1 showed no significant difference, m6A "readers" including YTHDF1 $(\mathrm{P}=0.002)$, YTHDF2 $(\mathrm{P}=0.009), \mathrm{YTHDF} 3(\mathrm{P}=0.002)$, HNRNPC $(\mathrm{P}<0.001)$ and HNRNPA2B1 $(\mathrm{P}=0.001)$ were significantly overexpressed in BRC tissues, consistent with the changes of their mRNA expression levels (Figure 4A and 4B). Furthermore, HPA analysis showed that YTHDC1 and HNRNPC were highly expressed in a pattern similar to the mRNA level changes in BRC tissues (Figure 5B and 5D). However, YTHDF2 and HNRNPA2B1 did not show prominent changes at the protein levels between BRC tissues and normal breast tissues (Figure 5A and 5C). The heterogeneity between the HPA data and our TMA cohort's results may be ascribed to the differences in test platforms. Moreover, YTHDF1 and YTHDF3 were absent from the HPA database. The results confirm m6A "readers" as highly altered molecules in BRC, indicating large variations in the expression of these genomic targets in BRC patients.

Furthermore, for the only two known m6A "eraser" demethylases, FTO and ALKBH5, we detected a much stronger expression of ALKBH5 $(\mathrm{P}<0.001)$ in $\mathrm{BRC}$ tissues compared with that in normal breast tissues, whereas FTO had no obvious changes (Figure 6A and 6B). IHC staining data from HPA database showed a predominant upregulation of ALKBH5 but downregulation of FTO in breast carcinoma tissues in comparison with normal breast tissues (Figure 6C and 6D).

\section{Subcellular location of m6A-associated genomic targets in BRC cell lines}

To explore the subcellular location of m6A-related genomic targets in BRC cell lines and their potential regulation mechanisms, immunofluorescence (IF) assay was performed on three different BRC cell lines (MDA-MB-231, MDA-MB-468 and MCF-7). As shown in Figure 7, there was strong nuclear staining as well as weak cytoplasmic staining for most of the m6A "writers" (WTAP, KIAA1429, METTL13, RBM15, and RBM15B) in the three BRC cell lines. However, the fluorescence signals of METTL14 and METTL16 in MDA-MB-231, MDA-MB-468 and MCF-7 were not significantly enhanced. For m6A "readers", IF staining showed that YTHDC1 was mainly located in the nucleus especially in MDA-MB-468. On the contrary, YTHDF1
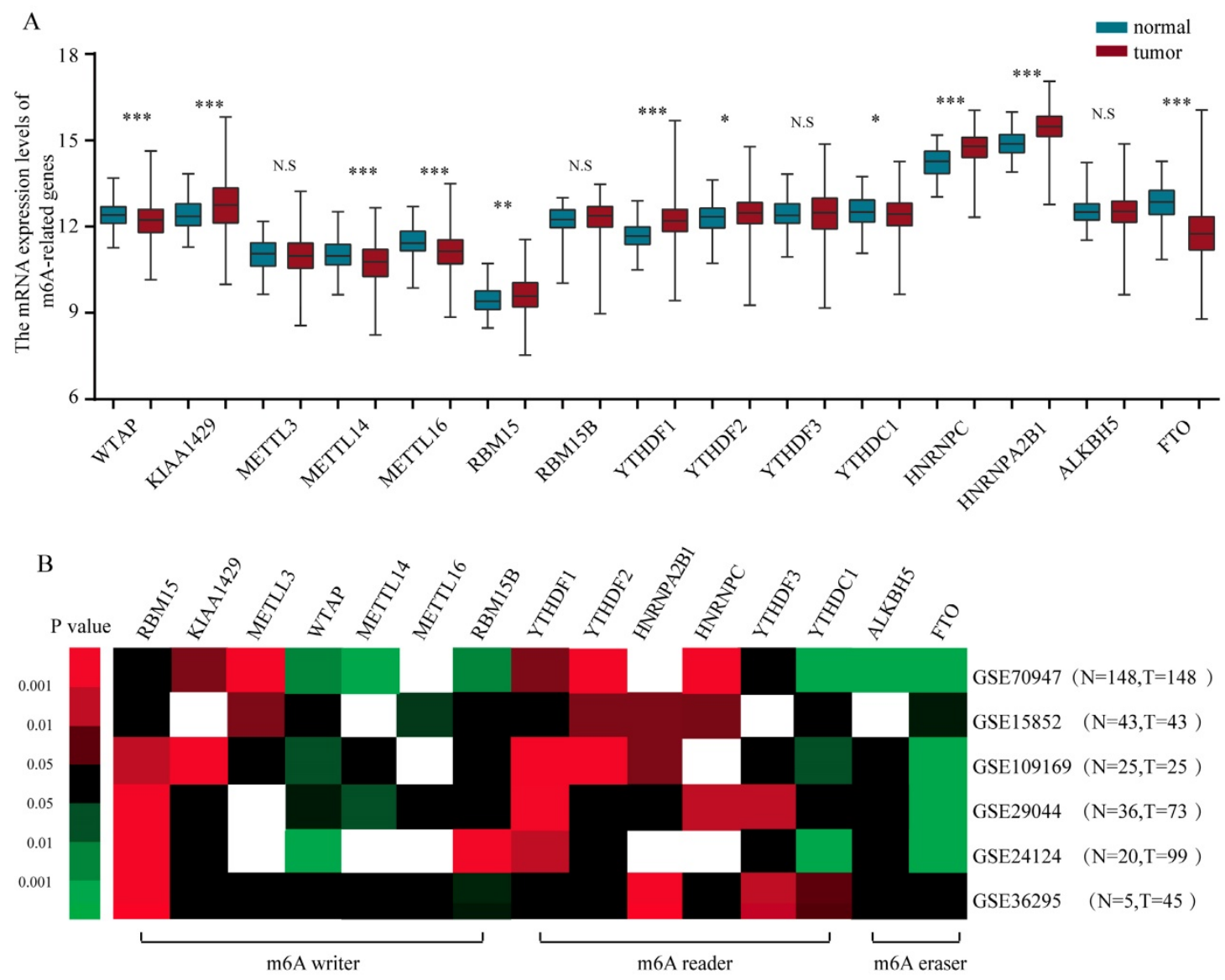

Figure 1. The altered expression profiles of m6A related genes in human BRC tissues. (A) The mRNA expression profiles of m6A-related genes in TCGA-BRCA cohort. (B) Heatmap showing the mRNA expression alteration of m6A related genomic targets in six independent GEO microarray datasets. Red means up-regulated; green means down-regulated; black means not significant; blank means genes are not expressed or absent in the datasets. Statistical analysis was performed in Student's $t$ test (unpaired, two-tailed) 
and YTHDF2 were mainly located in the cytoplasm, while HNRNPC was only detected in the nucleus (Figure 8A). YTHDF3 and HNRNPA2B1 were expressed both in the cytoplasm and nucleus (Figure 8A). We also detected fluorescence signals for ALKBH5 and FTO both in the cytoplasm and nucleus (Figure 8B). Furthermore, the expression intensity of FTO was weak in BRC cell lines, especially in MCF-7. These findings were similar to the IHC staining results in BRC tissues. The special subcellular location may further hint at a potential dynamic regulation of m6A in RNA processing, such as RNA decay, translation, splicing, transport and localization.

\section{The overexpression of YTHDF 1, YTHDF3 and KIAA1429 predicts poor survival in BRC patients}

To further investigate the potential correlation of differentially expressed m6A-related genomic targets to the clinical progression of BRC patients, we analyzed BRC patients' overall survival rates based on the data from TCGA-BRCA cohort. The results demonstrated that increased expressions of KIAA1429 ( $\mathrm{P}=0.032,95 \%$ CI: $1.03-1.96), \mathrm{YTHDF} 1$ ( $\mathrm{P}=0.049,95 \%$ CI: 1-1.91) and YTHDF3 (P<0.001, 95\% CI: 1.28-2.49) were significantly associated with poor overall survival rates in patients under the best cut-off value (Figure 9). Meanwhile, univariate and multivariate analyses showed that the increased expression of YTHDF3 was an independent predictor of cancer overall survival in patients with BRC (Table 2). Furthermore, we also explored whether these m6A-related genomic targets were associated with the relapse-free survival rate of patients. Similarly, we found that overexpression of YTHDF3 was correlated with lower relapse-free survival rate $(\mathrm{P}=0.016,95 \%$ $\mathrm{CI}=1.05-1.88$ ) (Figure 10).

\section{Discussion}

BRC is characterized by heterogeneity in which genetic or epigenetic factors play indispensable roles in its initiation and progression [17]. Currently, early diagnosis and precise individual therapy for BRC remain the greatest challenges. Therefore, to identify consistently altered genomic signatures is critical in BRC basic and clinical research. To uncover new therapeutic targets, we investigated the expression patterns of m6A-related genomic targets in BRC at the mRNA and protein levels. Through transcriptomic and proteomic analyses of m6A-associated genomic targets in large BRC cohorts, including RNA-seq data from public TCGA-BRCA and GEO microarray

A

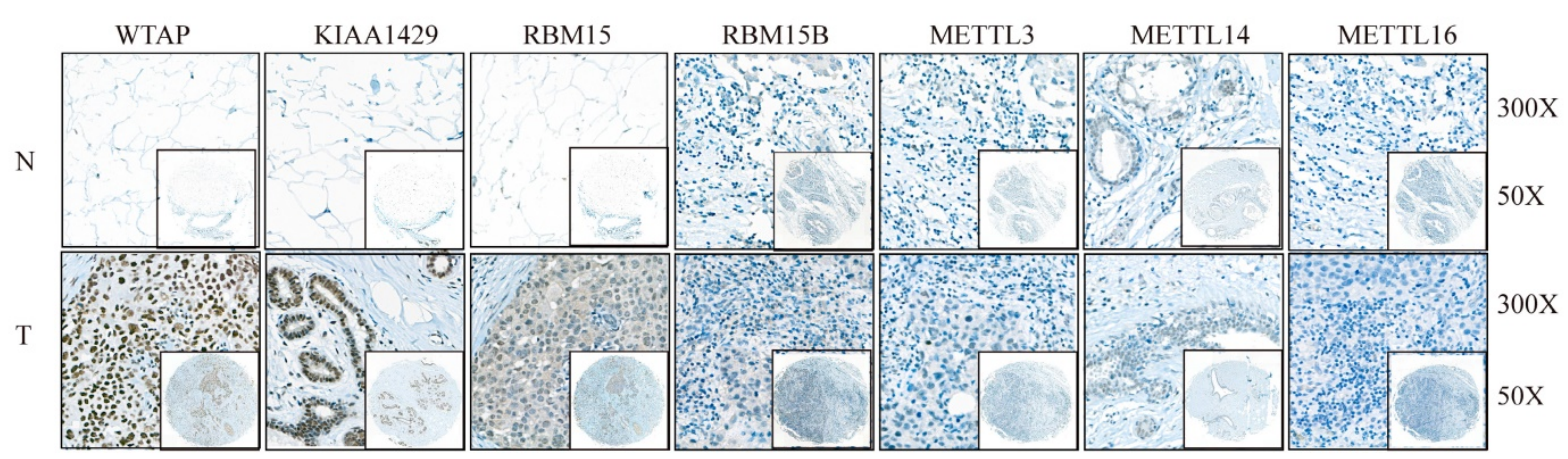

B

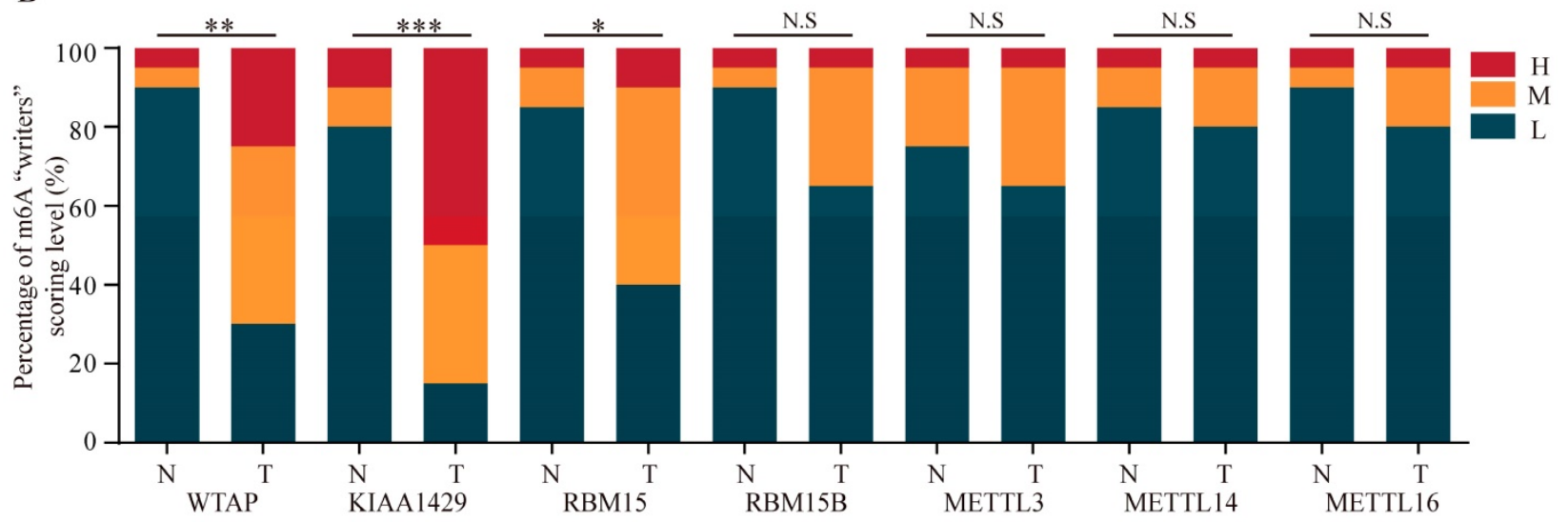

Figure 2. The protein levels of m6A "writers" are differentially expressed in BRC tissues and adjacent normal tissues. (A) Representative IHC staining result of m6A "writers" in BRC tissues (T) and the adjacent normal tissues (N) was shown. (B) The distribution of differentially expressed m6A "writers" in normal breast tissues (N) and breast cancer tissues $(T)$ was quantified. L, low expression; M, moderate expression; H, high expression; N.S, not significant; *P < 0.05 ; **P < 0.01 ; ***P< 0.001 . 
platforms as well as IHC staining data from the TMA cohorts, we observed that m6A-related genomic targets were frequently dysregulated in BRC and that the upregulation of YTHDF1, YTHDF3 and KIAA1429

is associated with poor patient survival.

Table 2. Independent prognostic factors for OS by multivariate analysis in TCGA BRCA cohort

\begin{tabular}{|c|c|c|c|c|c|c|c|}
\hline \multirow[t]{2}{*}{ Risk factors } & \multirow{2}{*}{$\begin{array}{l}\text { Clinicopathological } \\
\text { features }\end{array}$} & \multicolumn{3}{|c|}{ Univariate analysis } & \multicolumn{3}{|c|}{ Multivariate analysis } \\
\hline & & HR & $95 \%(\mathrm{CI})$ & $P$ value & HR & $95 \%(\mathrm{CI})$ & $P$ value \\
\hline \multirow[t]{2}{*}{ Age(years) } & $\leq$ median & 1.000 & $1.247-2.393$ & $0.001^{* *}$ & 1.000 & $1.350-2.675$ & $<0.001^{* *}$ \\
\hline & >median & 1.727 & & & 1.900 & & \\
\hline \multirow[t]{2}{*}{ Race } & White & 1.000 & $0.611-1.344$ & 0.625 & & & \\
\hline & Others & 0.906 & & & & & \\
\hline \multirow[t]{2}{*}{ Her-2 } & Positive & 1.000 & $0.929-2.108$ & 0.107 & & & \\
\hline & Negative & 1.399 & & & & & \\
\hline \multirow[t]{2}{*}{ TNM stage } & Stage I and II & 1.000 & $1.950-3.832$ & $<0.001^{* *}$ & 1.000 & $1.941-3.827$ & $<0.001^{* *}$ \\
\hline & Stage III and IV & 2.734 & & & 2.725 & & \\
\hline \multirow[t]{2}{*}{ KIAA1429 } & Low & 1.000 & & & 1.000 & & \\
\hline & High & 1.388 & $1.003-1.920$ & $0.048^{*}$ & 0.836 & $0.553-1.263$ & 0.394 \\
\hline \multirow[t]{2}{*}{ YTHDF1 } & Low & 1.000 & $1.000-1.920$ & $0.049^{*}$ & 1.000 & $0.924-1.924$ & 0.124 \\
\hline & High & 1.386 & & & 1.334 & & \\
\hline \multirow[t]{2}{*}{ YTHDF3 } & Low & 1.000 & $1.299-2.484$ & $<0.001^{* *}$ & 1.000 & $1.031-2.403$ & $0.036^{*}$ \\
\hline & High & 1.796 & & & 1.574 & & \\
\hline
\end{tabular}

Abbreviations: CI: confidential interval; HR: hazard ratio; TNM: tumor- node- metastasis. ${ }^{*} \mathrm{P}<.05,{ }^{* *} \mathrm{P}<.001$.

A

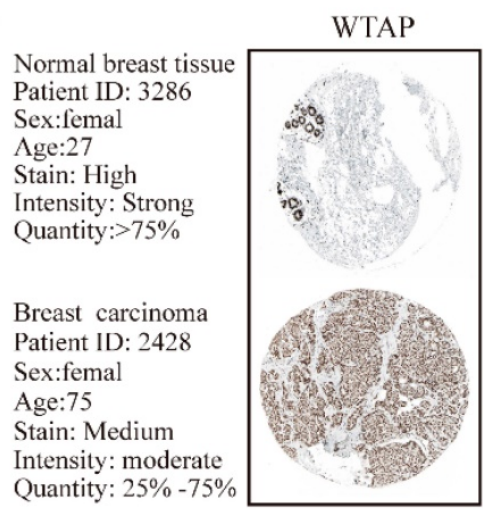

D

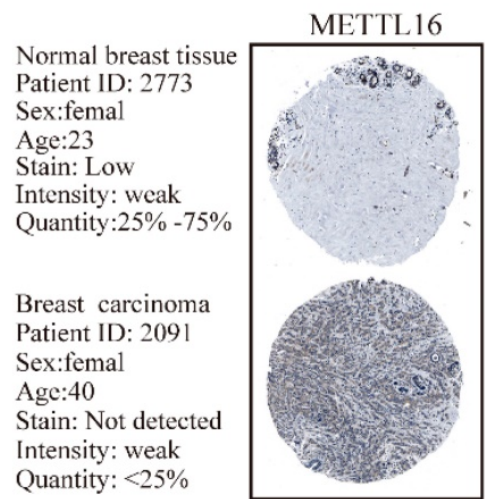

B

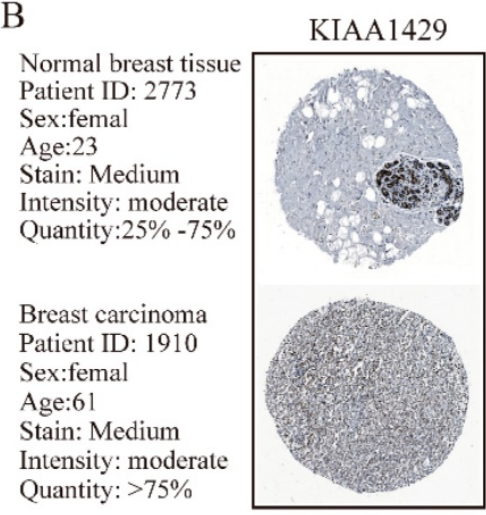

E
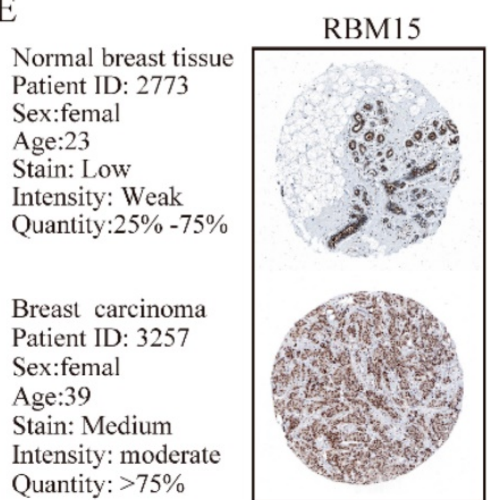

C

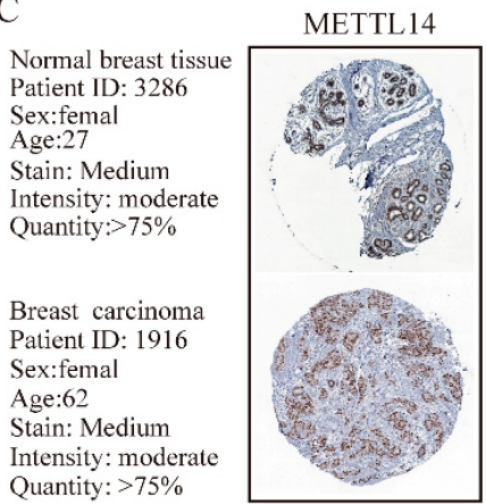

F

Normal breast tissue Patient ID: 3544

Sex:femal

Age:45

Stain: Low

Intensity: weak

Quantity:25\%-75\%

Breast carcinoma Patient ID: 1910

Sex:femal

Age:61

Stain: Not detected

Intensity: weak

Quantity: $<25 \%$
RBM15B

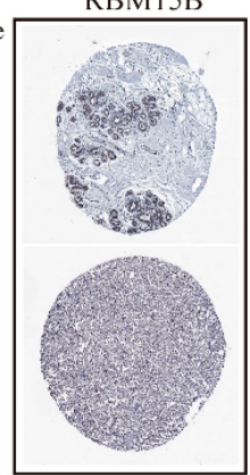

Figure 3. The protein expression levels of M6A “writers" in breast carcinoma tissues and normal breast tissues from HPA database are determined by IHC staining. (A) Representative IHC staining of WTAP in breast carcinoma and normal breast tissues. (B) Representative IHC staining of KIAA1429 in breast carcinoma and normal breast tissues. (C) Representative IHC staining of METTL14 in breast carcinoma and normal breast tissues. (D) Representative IHC staining of METTL16 in breast carcinoma and normal breast tissues. (E) Representative IHC staining of RBM15 in breast carcinoma and normal breast tissues. (F) Representative IHC staining of RBM15B in breast carcinoma and normal breast tissues. 
A

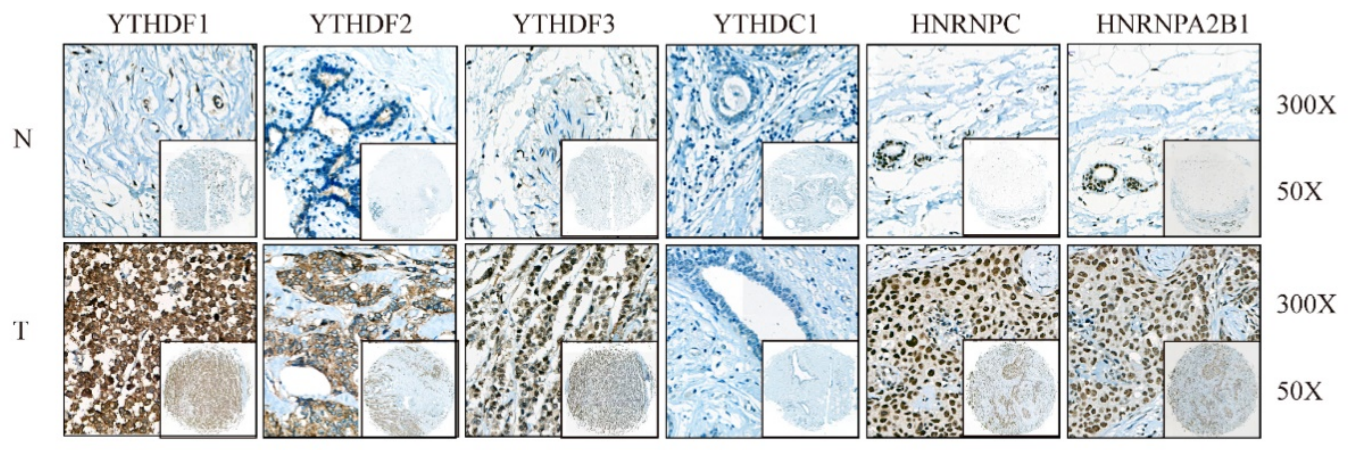

B

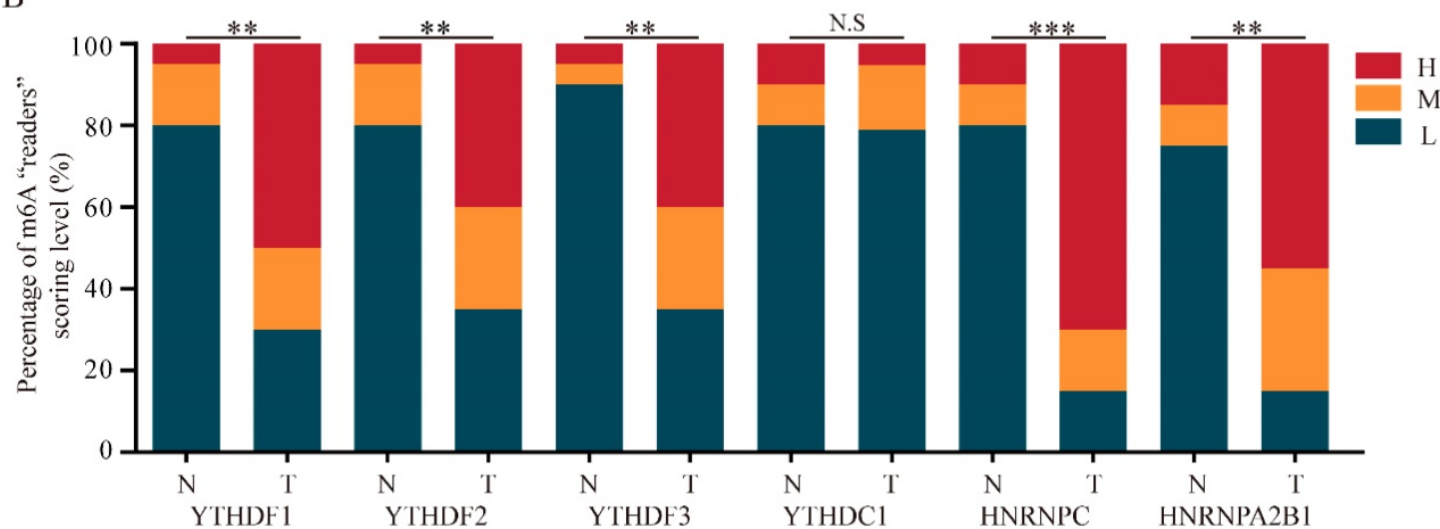

Figure 4. The protein levels of m6A "readers" are differentially expressed in BRC tissues and adjacent normal tissues. (A) Representative IHC staining result of m6A "readers" in BRC tissues $(\mathrm{T})$ and the adjacent normal tissues $(\mathrm{N})$ was shown. (B) The distribution of differentially expressed m6A "readers" in normal breast tissues (N) and breast cancer tissues $(\mathrm{T})$ was quantified. L, low expression; $\mathrm{M}$, moderate expression; $\mathrm{H}$, high expression; N.S, not significant; **P < 0.01 ; $* * * \mathrm{P}<0.001$.

A

Normal tissue
Patient ID: 3544
Sex:femal
Age: 45
Stain: Medium
Intensity: moderate
Quantity: $>75 \%$

Breast carcinoma
Patient ID: 830
Sex:femal
Age:24
Stain: Medium
Intensity: moderate
Quantity: $>75 \%$

C

Normal tissue
Patient ID: 2773
Sex:femal
Age: 23
Stain: High
Intensity: strong
Quantity: $>75 \%$

Breast carcinoma
Patient ID: 2428
Sex:femal
Age:39
Stain: High
Intensity: strong
Quantity: $>75 \%$

YTHDF2

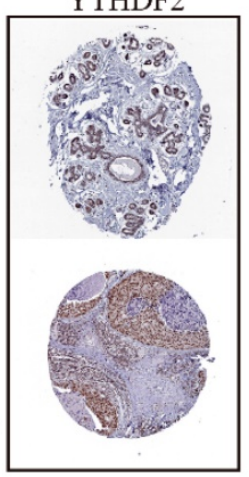

HNRNPA2B1

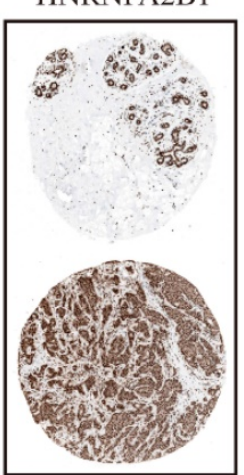

B

Normal tissue

Patient ID: 3544

Sex:femal

Age: 45

Stain: High

Intensity: strong

Quantity: $>75 \%$

Breast carcinoma Patient ID: 1939

Sex:femal

Age:87

Stain: Medium

Intensity: moderate

Quantity: $>75 \%$

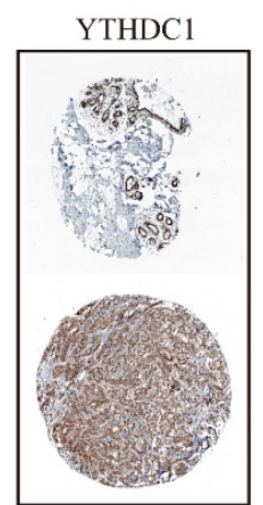

D

Normal tissue

Patient ID: 2104

Sex:femal

Age: 43

Stain: Medium

Intensity: moderate

Quantity:25\% -75\%

Breast carcinoma

Patient ID: 2428

Sex:femal

Age:75

Stain: High

Intensity: strong

Quantity: $>75 \%$

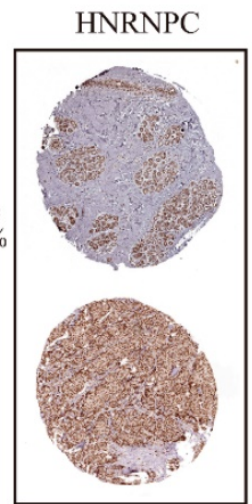

Figure 5. The protein expression levels of M6A "readers" in breast carcinoma tissues and normal breast tissues from HPA database are determined by IHC staining. (A) Representative IHC staining of YTHDF2 in breast carcinoma and normal breast tissues. (B) Representative IHC staining of YTHDC1 in breast carcinoma and normal breast tissues. (C) Representative IHC staining of HNRNPA2B1 in breast carcinoma and normal breast tissues. (D) Representative IHC staining of HNRNPC in breast carcinoma and normal breast tissues. 

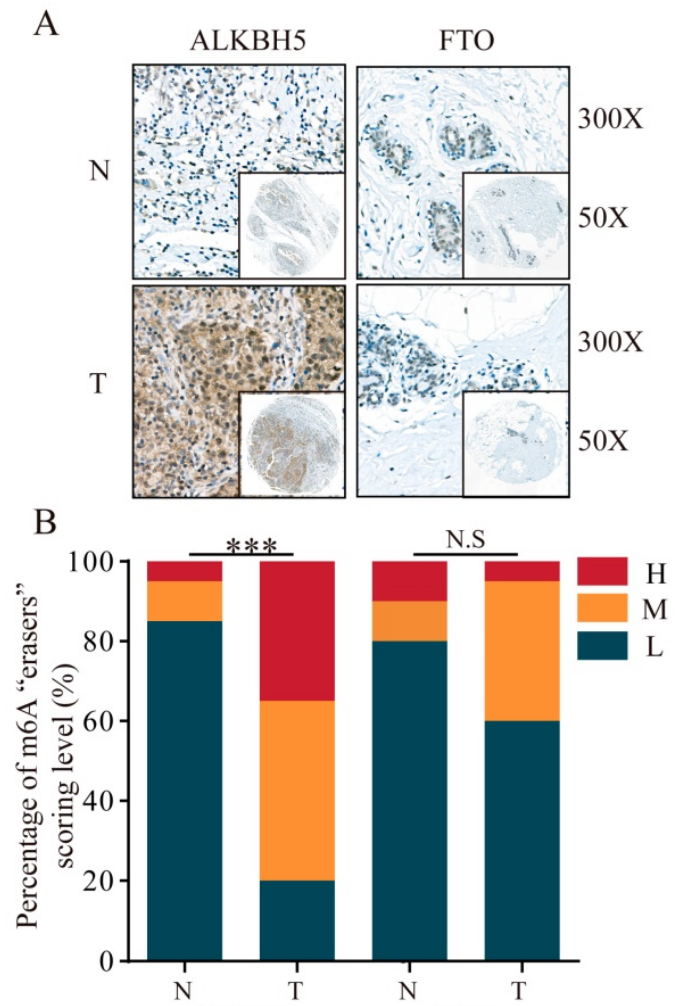

C

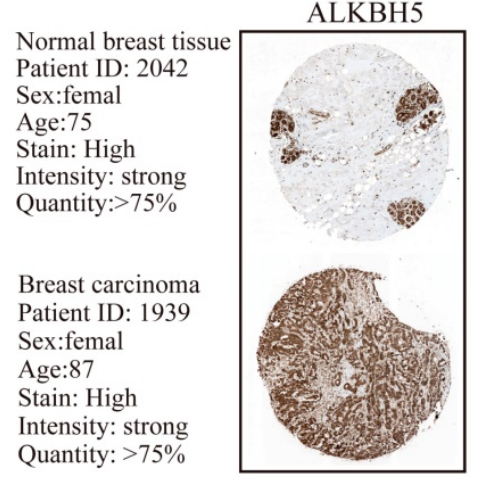

D

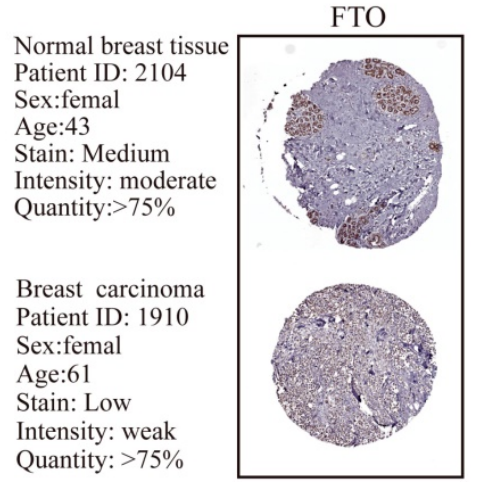

Figure 6. The protein levels of $m 6 A$ "erasers" are differentially expressed in BRC tissues and adjacent normal tissues. (A) Representative IHC staining result of m6A "erasers" in BRC tissues $(T)$ and the adjacent normal tissues $(N)$ was shown. (B) The distribution of differentially expressed m6A "erasers" in normal breast tissues $(N)$ and breast cancer tissues (T) was quantified. (C) Representative IHC staining of ALKBH5 in breast carcinoma and normal breast tissues from HPA database. (D) Representative IHC staining of FTO in breast carcinoma and normal breast tissues from HPA database. L, low expression; M, moderate expression; H, high expression; N.S, not significant. ***P< 0.001 .

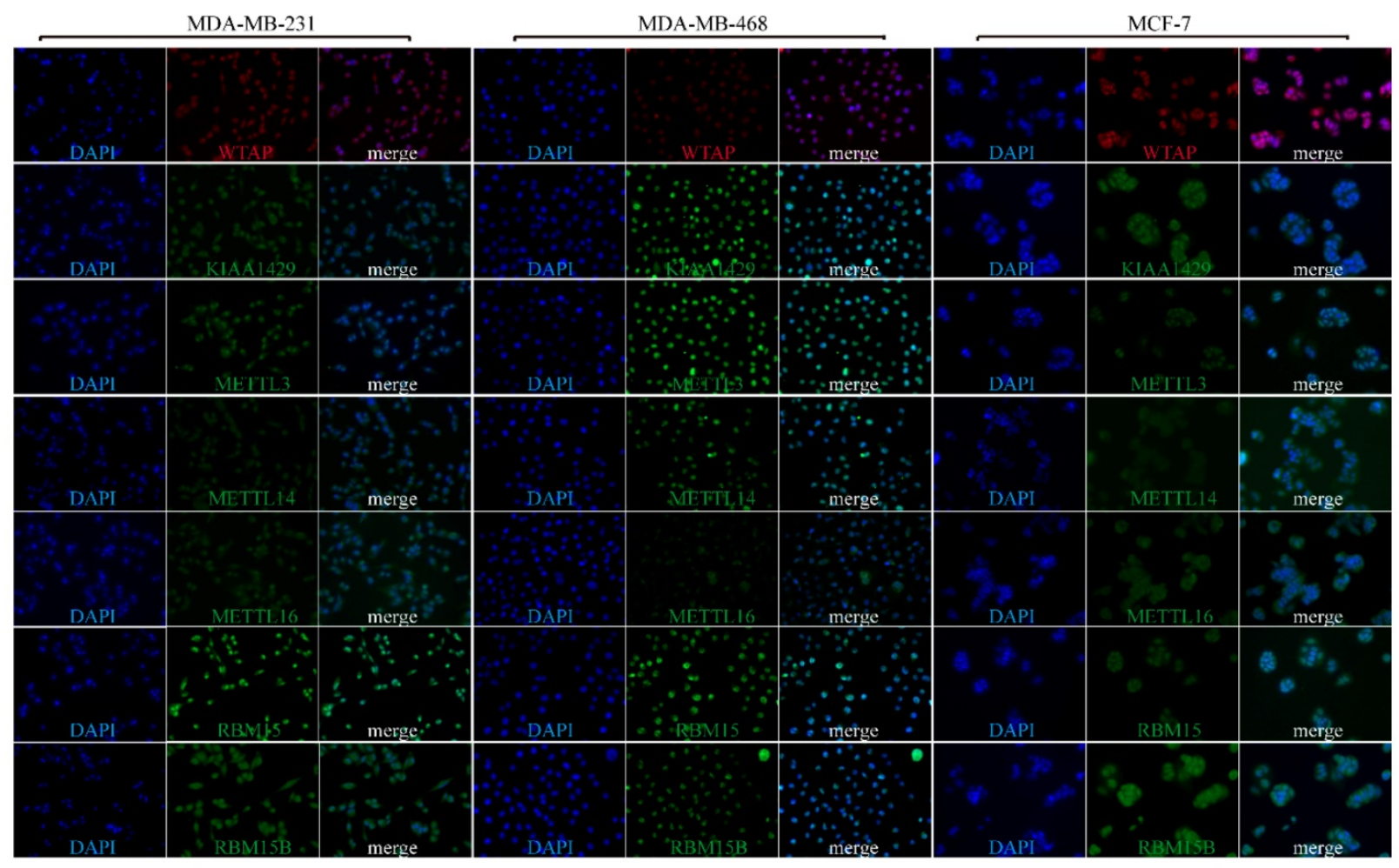

m6A writer

Figure 7. Subcellular location of m6A "writers" in three different BRC cell lines. MDA-MB-231, MDA-MB-468 and MCF-7 cells were stained by DAPI and different m6A "writers" and representative immunofluorescence photographs of m6A "writers" subcellular location in MDA-MB-231, MDA-MB-468 and MCF-7 cells were shown. 


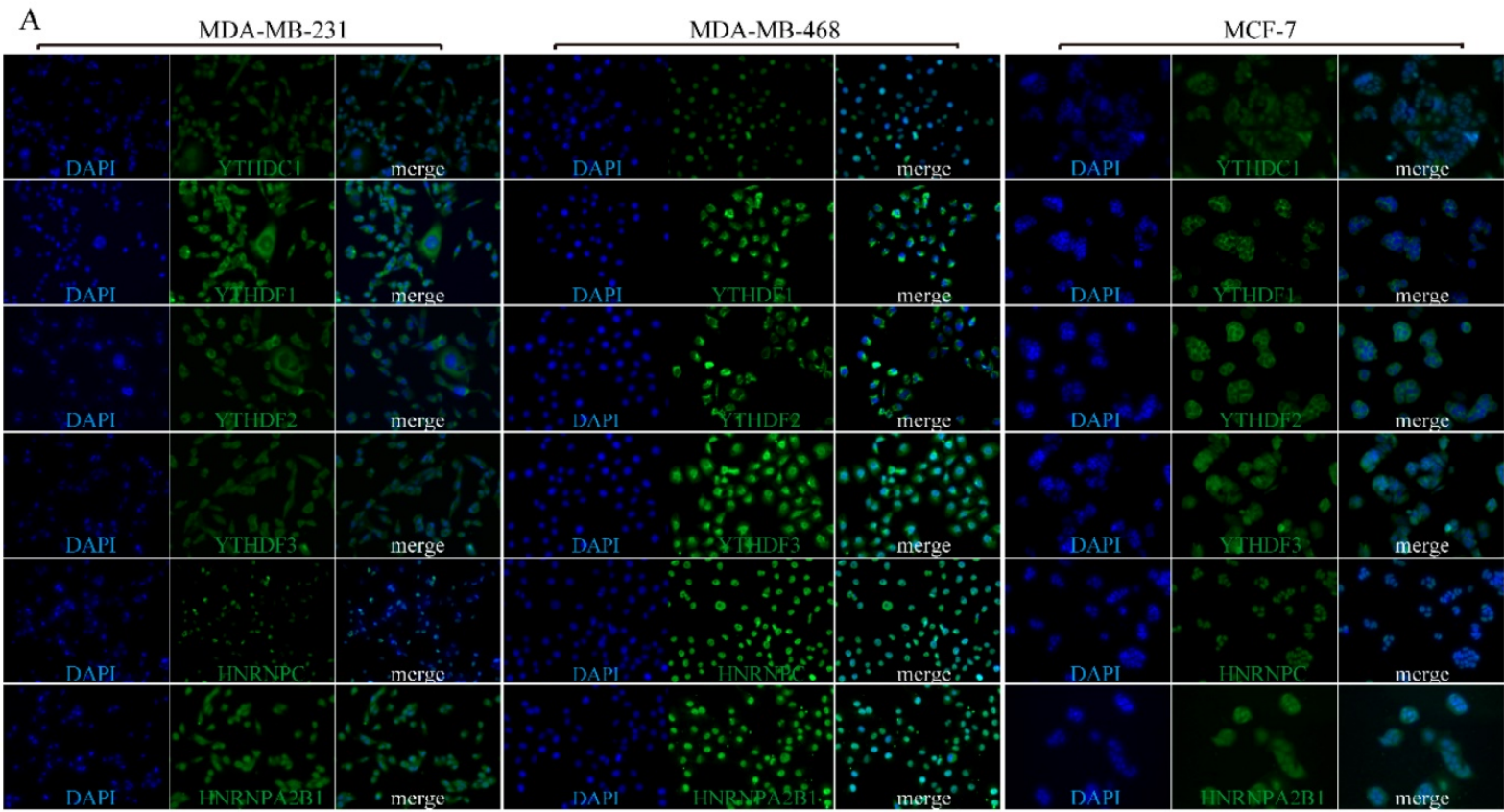

B

m6A reader

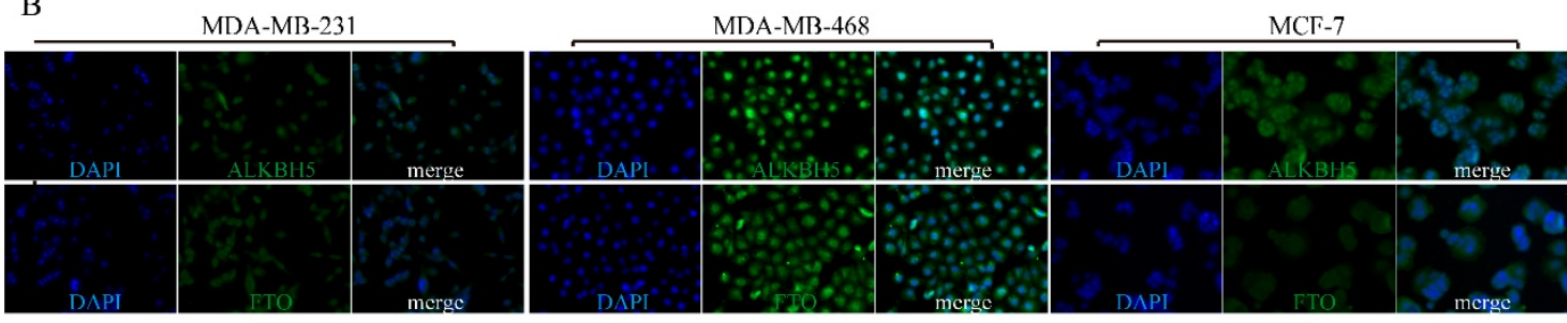

m6A eraser

Figure 8. Subcellular location of m6A "readers" and "erasers" in three different BRC cell lines. MDA-MB-231, MDA-MB-468 and MCF-7 cells were stained by DAPI and different m6A "readers" (A) or "erasers" (B) and representative immunofluorescence photographs of m6A "reader" or "erasers" subcellular location in MDA-MB-231, MDA-MB-468 and MCF-7 cells were shown.

As shown in Figure 11, m6A is generally considered to be installed by a large methyltransferase complex (m6A 'writers') that adds methylation modifications. WTAP, KIAA1429, METTL3, METTL14 and RBM15 are the core components of this complex [18]. Previously, the targetable genomic vulnerability of WTAP has been proposed in a wide spectrum of tumors, including cholangiocarcinoma [19], glioblastoma [20], acute myeloid leukaemia [21] and renal cell carcinoma [22]. In line with these studies, we found that WTAP was highly expressed at the protein level in BRC tissues, although it was not consistent with its mRNA level. This finding suggests an important role of WTAP in BRC. METTL3 was shown to participate in hepatocellular carcinoma (HCC) progression via mRNA m6A modification [23]. Additionally, RBM15 and METTL14 were demonstrated to play oncogenic roles in leukaemogenesis [24, 25]. These observations indicate that $\mathrm{m} 6 \mathrm{~A}$ dysregulation is a pervasive phenomenon in various malignancies. Similarly, our study observed significant upregulation of RBM15 and KIAA1429 as well as the downregulation of METTL14 and METTL16 in BRC tissues, which suggested that there might also be an abnormal N6-methyladenosine status in BRC.

M6A modification markers can be recognized by proteins, such as DF1, DF2, DF3 and DC1 that contain a specific YTH domain [26]. The DF family members (DF1, DF2, and DF3) are highly similar to each other and are predominantly cytoplasmic (Figure 11). Two additional members, HNRNPC and HNRNPA2B1, have been identified as nuclear m6A-binding proteins that affect alternative splicing of pre-mRNA and pre-microRNA [15, 27]. Previously, YTHDF1 was elucidated to be an oncogene in colorectal cancer [28]. YTHDF2 could participate in the progression of HCC and prostate cancer $[29,30]$. Moreover, HNRNPC was reported to control the aggressiveness of glioblastoma (GBM) cells by regulating PDCD4 [31]. In support of the role for m6A "readers" in various tumours, we also uncovered a higher abundance of "reader" proteins in BRC tissues. Thus, our study could help further identify m6A reader targets, except for 
YTHDC1, that are prone to be upregulated in BRC. Intriguingly, another study demonstrated that HNRNPC could contribute to crafting the BRC tumour microenvironment [32]. This finding suggests that N6-methyladenosine dysregulation commonly exists and plays a pivotal role in BRC development and progression.

FTO, one of the m6A "erasers" we found to be downregulated in BRC, has been reported to be critical for leukaemogenesis [33]. Notably, a previous study revealed that FTO single nucleotide polymorphisms (SNPs) were promising classifiers in predicting BRC risk, which indicates that FTO could function as a novel clinical biomarker [34]. Recently, the other demethylase, ALKBH5, was shown to potentiate tumourigenicity of glioblastoma by enhancing FOXM1 expression [35]. In our analysis, we also observed a significant up regulation of ALKBH5 at the protein level in BRC. Intriguingly, Zhang et al. identified ALKBH5-mediated modulation of RNA methylation or demethylation involving BRC cell stemness and pluripotency maintenance in a hypoxic environment $[36,37]$. This evidence strongly supports the fundamental mechanisms of $\mathrm{m} 6 \mathrm{~A}$ "erasers" in BRC with the induction of demethylation that has been linked to tumour growth and proliferation.

A writer

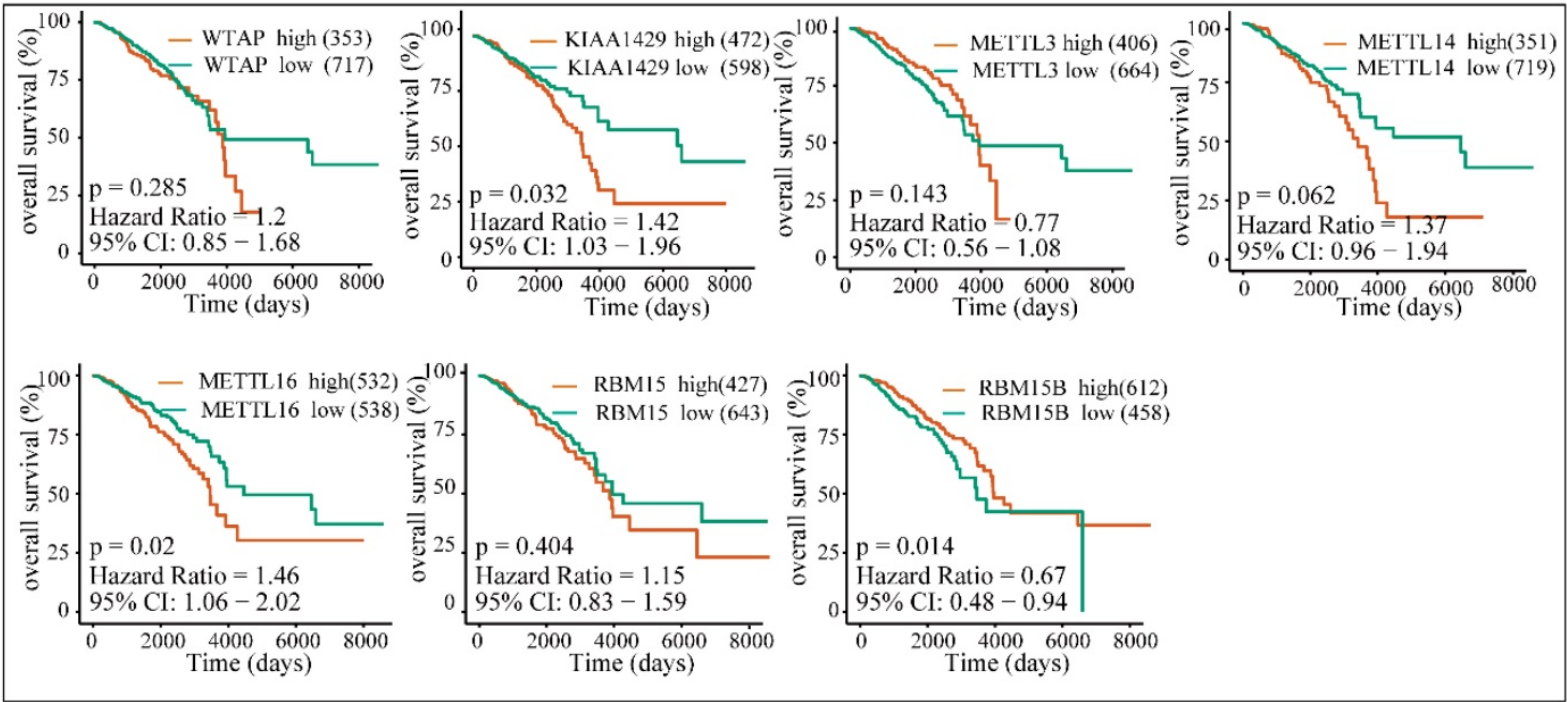

B reader
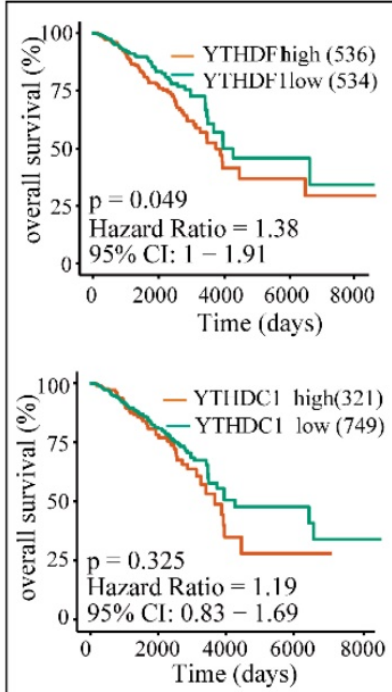
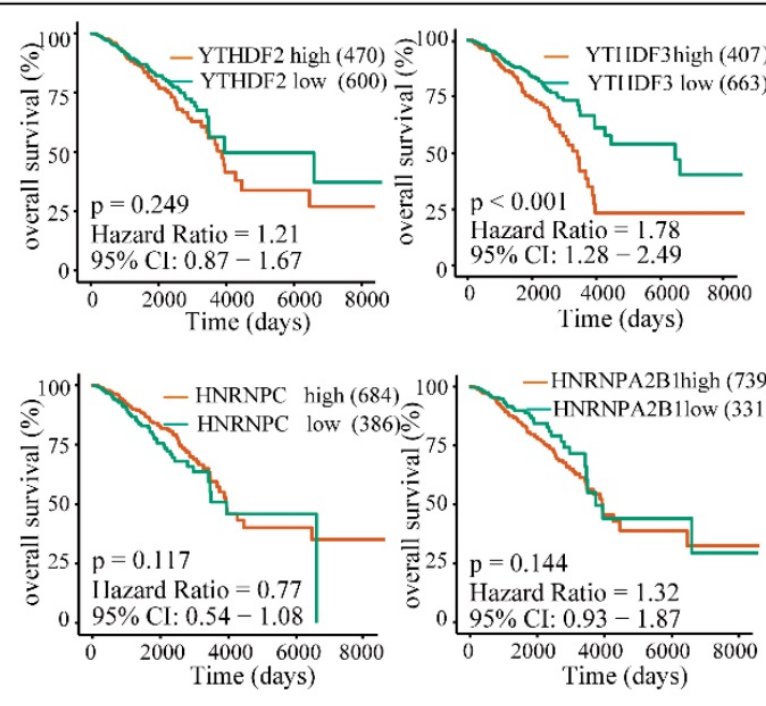

$\mathrm{C}$ eraser

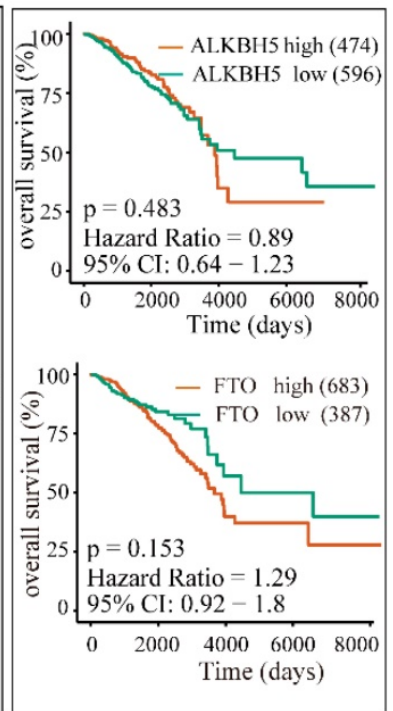

Figure 9. High expression of YTHDFI, YTHDF3 or KIAA1429 predicts poor prognosis in BRC patients. The correlations between the expression levels of m6A "writers" (A), "readers" (B), or "erasers" (C) and patients OS rates were examined by Kaplan-Meier analysis. 
A writer
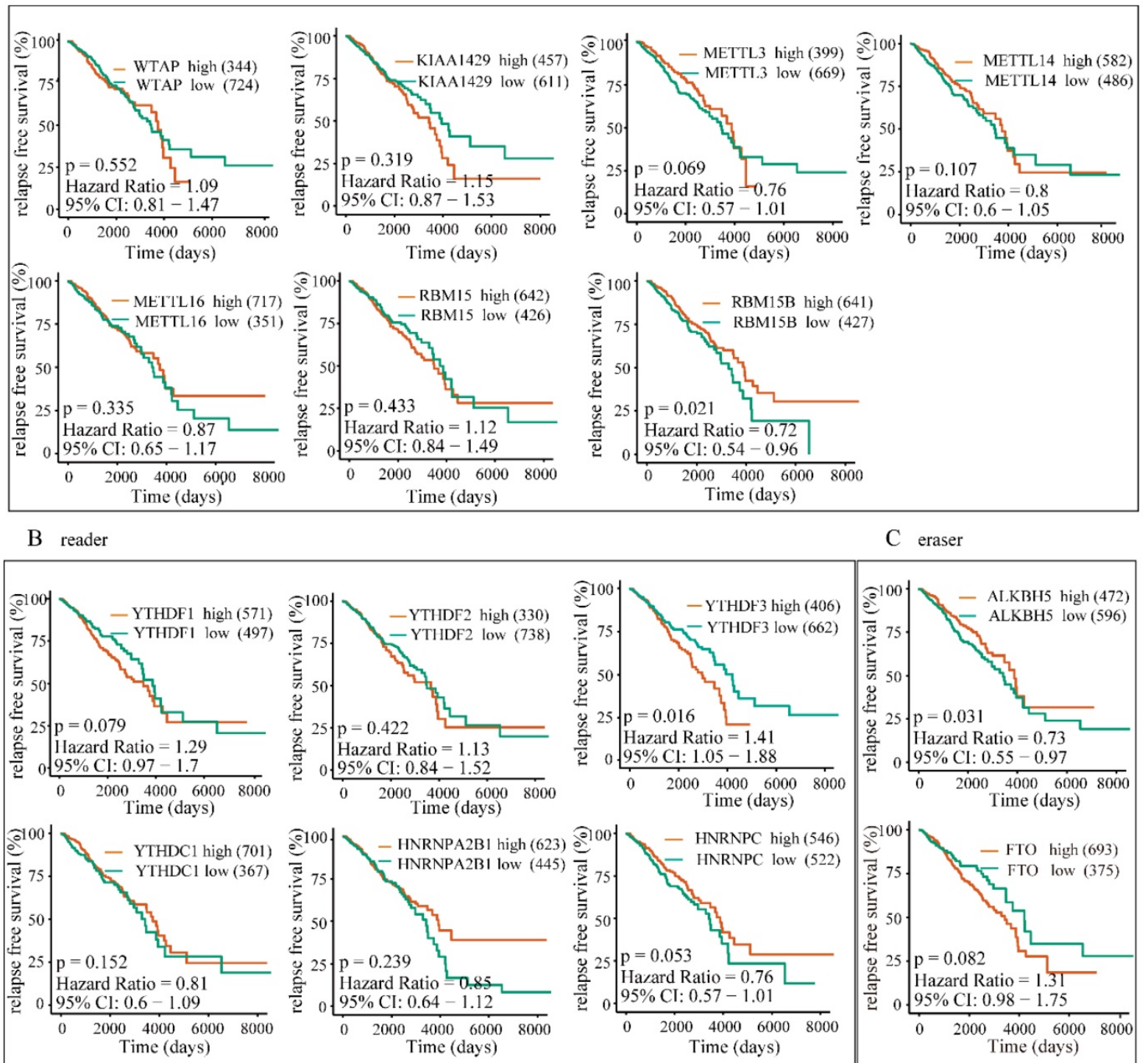

$\mathrm{C}$ eraser
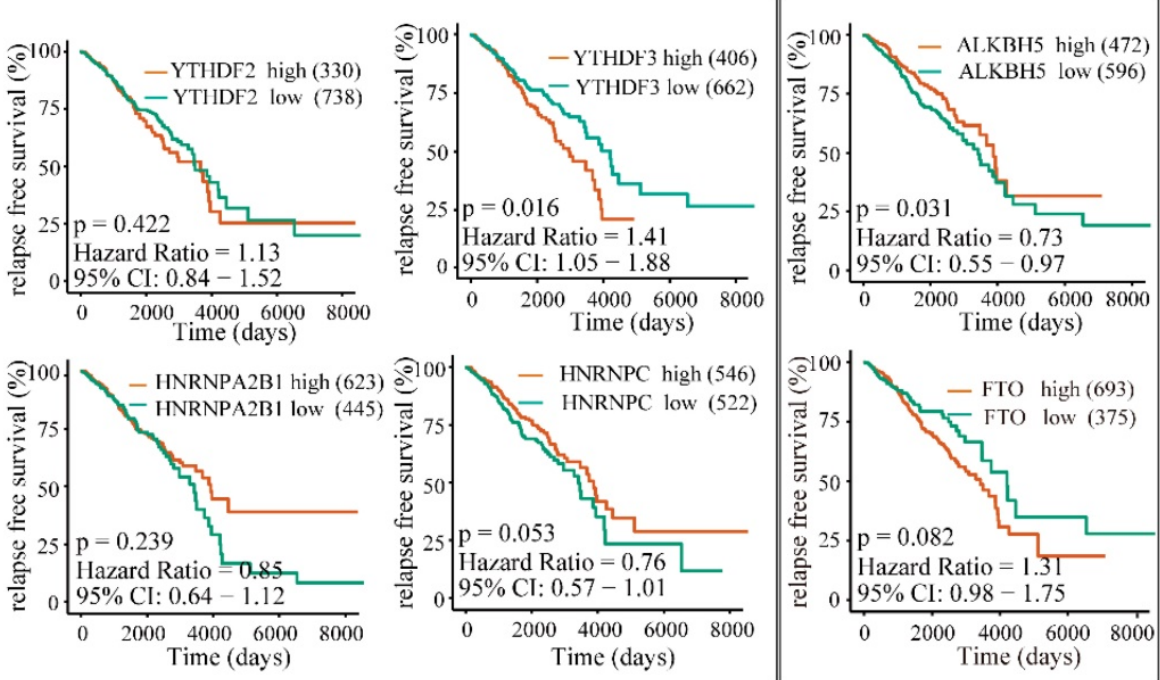

Figure 10. High expression of YTHDF3 is correlated with lower RFS rates. The correlations between the expression levels of m6A "writers" (A), "readers" (B), or "erasers" (C) and patients RFS rates were examined by Kaplan-Meier analysis.

In addition, based on clinical values and on our research, high expression levels of YTHDF1, YTHDF3 and KIAA1429 predicted unfavourable patient prognosis. Moreover, YTHDF3 expression levels are promising independent prognostic factors in BRC, which suggests that YTHDF3 could serve as a novel biomarker for BRC. In agreement with our study, m6A targets already described in the literature were proposed as biomarkers, prognostic indicators or therapeutic targets in cancer. Current research has reported that the elevated expression of YTHDF1 predicts a poor prognosis of HCC [38]. Moreover, HNRNPC has been identified as a candidate biomarker in gastric cancer chemoresistance [39]. Increased expression of FTO in leukaemia was believed to be associated with sensitivity to the chemotherapy drug R-2HG [40]. Therefore, it might be interesting to investigate the role of m6A-related genomic targets in drug resistance mechanisms in BRC. Altogether, in the context of personalized medicine, frequently altered targets could generate detectable mutation patterns in BRC and thus provide a rationale for the advancement of individualized interventions. Furthermore, genome-wide analysis is also a promising prospect for the research of m6A-specific drugs that target unique m6A-related gene patterns. In light of the deregulated m6A gene variability in different patients, exploring an individualized diagnostic and treatment regimen for BRC may be just around the corner.

\section{Conclusion}

M6A-related molecules are frequently dysregulated in BRC and associated with poor patient 


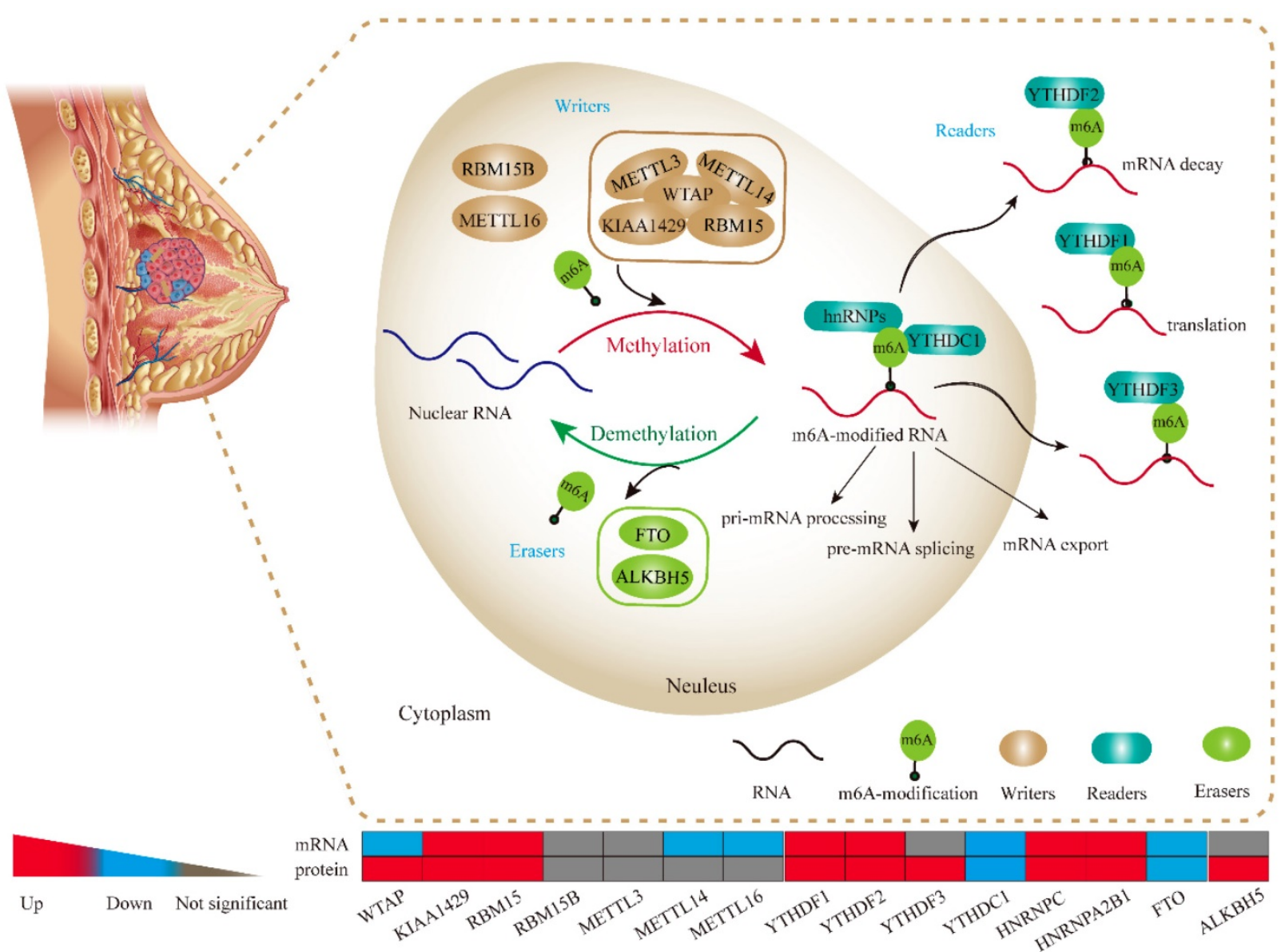

Figure 11. The potential molecular functions of m6A-based modification of RNA in BRC cell. m6A RNA methylation, known to be involved in all stages in the processing of RNA, is modulated by its "writers", "readers" and "erasers". m6A "writers" mainly refer to methylase complex (WTAP, KIAA1429, RBM15, METTL3 and METTL14), which add m6A modification on RNA, while m6A "readers" could serve as demethylases (FTO and ALKBH5). Meanwhile, both of "writers" and "erasers" dynamically fine-tune the m6A status in RNA within the nucleus. Readers (YTHDF1, YTHDF2, YTHDF3, YTHDC1, hnRNPs) are proteins that bind to m6A modifications and exert various functions including pre-mRNA splicing, pri-miRNA processing, nuclear export, RNA translation modulation and mRNA decay.

prognosis. In this study, we provided the first exposition of m6A-related genomic targets in BRC, and the results of our study may open novel avenues for future BRC studies in preclinical and clinical contexts.

\section{Abbreviations}

m6A: N6-methyladenosine; BRC: breast cancer; WTAP: Wilms' tumour 1-associated protein; TMA: tissue microarray; TCGA: The Cancer Genome Atlas; GEO: Gene Expression Omnibus; HPA: Human Protein Atlas database; OS: overall survival; RFS: relapse free survival.

\section{Supplementary Material}

Supplementary table.

http://www.jcancer.org/v10p5447s1.pdf

\section{Acknowledgements}

This study was approved by funds from the National Natural Science Foundation of China (81600506, and 81702757), the Medicine Science and
Technology Research Project of Henan Province (201702001), the Youth innovation fund of the First Affiliated Hospital of Zhengzhou University (YNQN2017032 and YNQN2017031). The funding body had no role in the design of the study, or in the manuscript writing.

\section{Ethical statement}

The project of this study was in accordance with the ethical standards of the Declaration of Helsinki 1975 and with the principles of institutional and national research committee.

\section{Competing Interests}

The authors have declared that no competing interest exists.

\section{References}

1. Larsen MJ, Thomassen M, Gerdes AM, Kruse TA. Hereditary breast cancer: clinical, pathological and molecular characteristics. Breast Cancer (Auckl). 2014; 8: 145-55.

2. Garrido-Castro AC, Lin NU, Polyak K. Insights into Molecular Classifications of Triple-Negative Breast Cancer: Improving Patient Selection for Treatment. Cancer Discovery. 2019; 9: 176-98. 
3. Bray F, Ferlay J, Soerjomataram I, Siegel RL, Torre LA, Jemal A. Global cancer statistics 2018: GLOBOCAN estimates of incidence and mortality worldwide for 36 cancers in 185 countries. CA: a cancer journal for clinicians. 2018; 68: 394-424.

4. Muntean AG, Hess JL. Epigenetic dysregulation in cancer. Am J Pathol. 2009; 175: $1353-61$.

5. Lai AY, Wade PA. Cancer biology and NuRD: a multifaceted chromatin remodelling complex. Nature Reviews Cancer. 2011; 11: 588-96.

6. Akhavan-Niaki, Haleh, Samadani, Akbar A. DNA Methylation and Cancer Development: Molecular Mechanism. Cell Biochemistry \& Biophysics. 2013; 67: 501-13.

7. Berger SL. Histone modifications in transcriptional regulation. Current Opinion in Genetics \& Development. 2002; 12: 142-8.

8. Roignant JY, Soller M. m(6)A in mRNA: An Ancient Mechanism for Fine-Tuning Gene Expression. Trends in genetics : TIG. 2017; 33: 380-90.

9. Wojtas MN, Pandey RR, Mendel M, Homolka D, Sachidanandam R, Pillai RS. Regulation of m(6)A Transcripts by the $3^{\prime}-->5$ ' RNA Helicase YTHDC2 Is Essential for a Successful Meiotic Program in the Mammalian Germline. Molecular cell. 2017; 68: 374-87.

10. Lence T, Akhtar J, Bayer M, Schmid K, Spindler L, Ho $\mathrm{CH}$, et al. m6A modulates neuronal functions and sex determination in Drosophila. Nature. 2016; 540: 242-7.

11. Du K, Zhang L, Lee T, Sun T. m6A RNA Methylation Controls Neural Development and Is Involved in Human Diseases. Molecular Neurobiology. 2018: 1-11.

12. Wang CY, Yeh JK, Shie SS, Hsieh IC, Wen MS. Circadian rhythm of RNA N6-methyladenosine and the role of cryptochrome. Biochemical \& Biophysical Research Communications. 2015; 465: 88-94.

13. Zhou J, Wan J, Shu XE, Mao Y, Liu XM, Yuan X, et al. N(6)-Methyladenosine Guides mRNA Alternative Translation during Integrated Stress Response. Molecular cell. 2018; 69: 636-47.

14. Chen J, Sun $Y, X u X$, Wang D, He J, Zhou H, et al. YTH domain family 2 orchestrates epithelial-mesenchymal transition/proliferation dichotomy in pancreatic cancer cells. Cell cycle. 2017; 16: 2259-71.

15. Pan $\mathrm{Y}, \mathrm{Ma} \mathrm{P}$, Liu $\mathrm{Y}, \mathrm{Li} \mathrm{W}$, Shu $\mathrm{Y}$. Multiple functions of $\mathrm{m}(6) \mathrm{A}$ RNA methylation in cancer. Journal of hematology \& oncology. 2018; 11: 48-58.

16. Jaffrey SR, Kharas MG. Emerging links between m(6)A and misregulated mRNA methylation in cancer. Genome medicine. 2017; 9: 2-4.

17. Pasculli B, Barbano R, Parrella P. Epigenetics of breast cancer: Biology and clinical implication in the era of precision medicine. Seminars in cancer biology. 2018; 51: 22-35.

18. Years IR. Rethinking m6A Readers, Writers, and Erasers. Annual Review of Cell \& Developmental Biology. 2017; 33: 319-42.

19. Jo HJ, Shim HE, Han ME, Kim HJ, Kim KS, Baek S, et al. WTAP regulates migration and invasion of cholangiocarcinoma cells. Journal of Gastroenterology. 2013; 48: 1271-82.

20. Du-Il J, Weon LS, Myoung-Eun H, Hyun-Jung K, Seon-Ae S, Gi-Yeong H, et al. Expression and roles of Wilms' tumor 1-associating protein in glioblastoma. Cancer science. 2012; 103: 2102-9.

21. Bansal H, Yihua Q, Iyer SP, Ganapathy S, Proia DA, Proia D, et al. WTAP is a novel oncogenic protein in acute myeloid leukemia. Leukemia. 2014; 28: 1171-4.

22. Tang J, Wang F, Cheng G, Si S, Sun X, Han J, et al. Wilms' tumor 1-associating protein promotes renal cell carcinoma proliferation by regulating CDK2 mRNA stability. Journal of experimental \& clinical cancer research : CR. 2018; 37: 40-51.

23. Chen M, Wei L, Law CT, Tsang FH, Shen J, Cheng CL, et al. RNA N6-methyladenosine methyltransferase-like 3 promotes liver cancer progression through YTHDF2-dependent posttranscriptional silencing of SOCS2. Hepatology. 2018; 67: 2254-70

24. Weng H, Huang H, Wu H, Qin X, Zhao BS, Dong L, et al. METTL14 Inhibits Hematopoietic Stem/Progenitor Differentiation and Promotes Leukemogenesis via mRNA m 6 A Modification. Cell Stem Cell. 2018; 22: 191-205.

25. Zhang L, Tran NT, Su H, Wang R, Lu Y, Tang H, et al. Cross-talk between PRMT1-mediated methylation and ubiquitylation on RBM15 controls RNA splicing. Elife. 2015; 4.

26. Liao S, Sun H, Xu C. YTH Domain: A Family of N(6)-methyladenosine (m(6)A) Readers. Genomics, proteomics \& bioinformatics. 2018; 16: 99-107.

27. Alarcón CR, Hani G, Hyeseung L, Xuhang L, Saeed T, Tavazoie SF. HNRNPA2B1 Is a Mediator of m(6)A-Dependent Nuclear RNA Processing Events. Cell. 2015; 162: 1299-308.

28. Nishizawa Y, Konno M, Asai A, Koseki J, Kawamoto K, Miyoshi N, et al. Oncogene c-Myc promotes epitranscriptome m6A reader YTHDF1 expression in colorectal cancer. Oncotarget. 2018; 9: 7476-86.

29. Zhong L, Liao D, Zhang M, Zeng C, Li X, Zhang R, et al. YTHDF2 suppresses cell proliferation and growth via destabilizing the EGFR mRNA in hepatocellular carcinoma. Cancer letters. 2019; 442: 252-61.

30. Li J, Meng $S, X_{u} M$, Wang $S$, He L, Xu X et al. Downregulation of N6-methyladenosine binding YTHDF2 protein mediated by miR-493-3p suppresses prostate cancer by elevating N6-methyladenosine levels. Oncotarget. 2018; 9: 3752-64.

31. Park YM, Hwang SJ, Masuda K, Choi KM, Jeong MR, Nam DH, et al, Heterogeneous nuclear ribonucleoprotein $\mathrm{C} 1 / \mathrm{C} 2$ controls the metastatic potential of glioblastoma by regulating PDCD4. Molecular and cellular biology. 2012; 32: 4237-44

32. Wu Y, Zhao W, Liu Y, Tan X, Li X, Zou Q, et al. Function of HNRNPC in breast cancer cells by controlling the dsRNA-induced interferon response. The EMBO journal. 2018; 37:17-35

33. Li Z, Weng H, Su R, Weng X, Zuo Z, Li C, et al. FTO Plays an Oncogenic Role in Acute Myeloid Leukemia as a N(6)-Methyladenosine RNA Demethylase. Cancer cell. 2017; 31: 127-41.

34. Kaklamani V, Yi N, Sadim M, Siziopikou K, Zhang K, Xu Y, et al. The role of the fat mass and obesity associated gene (FTO) in breast cancer risk. BMC medical genetics. 2011; 12: 52-62.

35. Zhang S, Zhao BS, Zhou A, Lin K, Zheng S, Lu Z, et al. m(6)A Demethylase ALKBH5 Maintains Tumorigenicity of Glioblastoma Stem-like Cells by Sustaining FOXM1 Expression and Cell Proliferation Program. Cancer cell. 2017; 31: 591-606.

36. Zhang C, Samanta D, Lu H, Bullen JW, Zhang H, Chen I, et al. Hypoxia induces the breast cancer stem cell phenotype by HIF-dependent and ALKBH5-mediated m(6)A-demethylation of NANOG mRNA. Proceedings of the National Academy of Sciences of the United States of America. 2016; 113: 2047-56.

37. Zhang C, Zhi WI, Lu H, Samanta D, Chen I, Gabrielson E, et al. Hypoxia-inducible factors regulate pluripotency factor expression by ZNF217and ALKBH5-mediated modulation of RNA methylation in breast cancer cells. Oncotarget. 2016; 7: 64527-42.

38. Zhao X, Chen $Y$, Mao Q, Jiang X, Jiang W, Chen J, et al. Overexpression of YTHDF1 is associated with poor prognosis in patients with hepatocellular carcinoma. Cancer Biomarkers. 2018; 21: 859-68.

39. Huang H, Han Y, Zhang C, Wu J, Feng J, Qu L, et al. HNRNPC as a candidate biomarker for chemoresistance in gastric cancer. Tumour biology : the journal of the International Society for Oncodevelopmental Biology and Medicine. 2016; 37: 3527-34

40. Su R, Dong L, Li C, Nachtergaele S, Wunderlich M, Qing Y, et al. R-2HG Exhibits Anti-tumor Activity by Targeting FTO/m 6 A/MYC/CEBPA Signaling. Cell. 2018; 172: 90-105. 